\title{
Bisperoxovanadium promotes motor neuron survival and neuromuscular innervation in amyotrophic lateral sclerosis
}

\author{
Junmei Wang ${ }^{1}$, Lydia Tierney', Ranjeet Mann' ${ }^{1}$, Thomas Lonsway ${ }^{1}$ and Chandler L. Walker ${ }^{1,2^{*}}$
}

\begin{abstract}
Amyotrophic lateral sclerosis (ALS) is the most common motor neuron (MN) disease, with no present cure. The progressive loss of MNs is the hallmark of ALS. We have previously shown the therapeutic effects of the phosphatase and tensin homolog (PTEN) inhibitor, potassium bisperoxo (picolinato) vanadium (bpV[pic]), in models of neurological injury and demonstrated significant neuroprotective effects on MN survival. However, accumulating evidence suggests PTEN is detrimental for MN survival in ALS. Therefore, we hypothesized that treating the mutant superoxide dismutase $1 \mathrm{G} 93 \mathrm{~A}$ ( $\mathrm{mSOD}^{\mathrm{G}}{ }^{\mathrm{G} 3 \mathrm{~A}}$ ) mouse model of ALS during motor neuron degeneration and an in vitro model of $\mathrm{mSOD} 1^{\mathrm{G} 93 \mathrm{~A}}$ motor neuron injury with bpV(pic) would prevent motor neuron loss. To test our hypothesis, we treated mSOD $1{ }^{\mathrm{G} 93 \mathrm{~A}}$ mice intraperitoneally daily with $400 \mu \mathrm{g} / \mathrm{kg} \mathrm{bpV}$ (pic) from 70 to 90 days of age. Immunolabeled MNs and microglial reactivity were analyzed in lumbar spinal cord tissue, and bpV(pic) treatment significantly ameliorated ventral horn motor neuron loss in $\mathrm{mSOD} 1^{\mathrm{G} 93 \mathrm{~A}}$ mice $(p=0.003)$ while not significantly altering microglial reactivity $(p=0.701)$. Treatment with bpV(pic) also significantly increased neuromuscular innervation ( $p=0.018)$ but did not affect muscle atrophy. We also cultured motor neuron-like NSC-34 cells transfected with a plasmid to overexpress mutant SOD $1^{\mathrm{G} 93 \mathrm{~A}}$ and starved them in serum-free medium for $24 \mathrm{~h}$ with and without bpV(pic) and downstream inhibitor of Akt signaling, LY294002. In vitro, bpV(pic) improved neuronal viability, and Akt inhibition reversed this protective effect $(p<0.05)$. In conclusion, our study indicates systemic bpV(pic) treatment could be a valuable neuroprotective therapy for ALS.
\end{abstract}

Keywords: Amyotrophic lateral sclerosis, ALS, PTEN, bpV, Akt, Motor neuron

Amyotrophic lateral sclerosis (ALS) is a devastating fatal neurological disease without a cure. Progressive upper and lower motor neuron (MN) death in the brain and spinal cord are hallmarks of the disease, leading to muscle paralysis and, eventually, respiratory failure. The average prognosis for survival is $3-5$ years following diagnosis [1]. Research into the mechanisms of MN loss and methods to promote their survival have been key areas of study for many years, though no truly effective treatments have

*Correspondence: chalwalk@iu.edu

${ }^{1}$ Department of Biomedical Sciences and Comprehensive Care, Indiana University School of Dentistry, Indianapolis, IN 46202, USA

Full list of author information is available at the end of the article been discovered. Although ALS is a non-cell-autonomous disease with many dynamic factors influencing $\mathrm{MN}$ degeneration, intrinsic mechanisms within the MN are ultimately essential in the process. Evidence over the last several years has highlighted the negative influence of the phosphatase and tensin homolog (PTEN), an antagonist of phosphatidylinositol-3-kinase (PI3K)/Akt signaling on MN vitality in ALS and other MN diseases [2-4]. This is in line with evidence in other neurological diseases and disorders, making PTEN a potentially prime target for therapeutic intervention.

We have previously demonstrated potent neuroprotective effects of PTEN inhibition on MN survival in 
central nervous system injury models. Systemic treatment with the PTEN antagonist small molecule, potassium bisperoxo (picolinato) vanadium (bpV[pic]) during neurodegenerative phases post-injury reduced the extent of neurological damage, enhanced ventral horn MN survival, and improved vascularization around the site of injury [5]. In addition, our findings demonstrated that $\mathrm{bpV}$ (pic) reduced acute neuronal atrophy and upregulated neuronal Akt signaling as a mechanism of its neuroprotective effects $[6,7]$. With growing data in support of $\mathrm{bpV}$ (pic) as a neuroprotective therapy and accumulating evidence that PTEN may be contributing to MN degeneration in ALS, we proposed to test this therapy in animal and in vitro models of ALS and determine whether similar protective effects are mediated.

Motor neuron degeneration is evident pre-symptomatically between 60 and 90 days of age in the gold standard mutant superoxide dismutase 1 G93A (mSOD1 ${ }^{\text {G93A }}$ ) mouse model of ALS (B6.Cg$\mathrm{Tg}$ (SOD1*G93A)1Gur/J \#004435; Jackson Laboratory) [8]. In the present study, we administered $400 \mu \mathrm{g} /$ $\mathrm{kg}$ bpV(pic) (Cat. No. SML0885, Sigma-Aldrich) $(\mathrm{n}=7)$ or saline (vehicle) daily (intraperitoneal, i.p.) $(n=7)$ in $\mathrm{mSOD} 1^{\mathrm{G} 93 \mathrm{~A}}$ mice from 70 to 90 days of age followed by euthanasia via i.p. injection of 2,2,2-tribromoethanol (Avertin) (Cat. No. T48402, Millipore Sigma) and transcardial perfusion with $0.1 \mathrm{M}$ phosphate-buffered saline (PBS) and 4\% paraformaldehyde (PFA) in PBS. Wild-type littermates were also euthanized for control tissue isolation and preparation $(n=6)$. The lumbar spinal cord was harvested, cryosectioned cross-sectionally at $20 \mu \mathrm{m}$, and serially mounted on microscope slides (Superfrost Plus, Fisher Scientific). Selected lumbar spinal cord sections were incubated with primary rabbit monoclonal antibody against the neuronal nuclear marker, NeuN (1:500, Cat. No. 24307, Cell Signaling, Inc.) and others with rabbit polyclonal Iba-1 antibody (1:1000, Cat. No. 019-19741, Wako Puro Chemical Corp.) overnight for microglial labeling. Fluorophore-conjugated secondary antibody (Jackson Immunoresearch) was applied the following day, and images of the ventral horn were taken of the labeled sections using a Nikon Eclipse Ti epifluorescent microscope and Nikon NIS-Elements software. NeuNpositive neurons in the ventral horn were counted, and the intensity of Iba- 1 immunofluorescence was measured in Image J software (NIH).

As neuromuscular junction denervation and atrophy begin prior to symptom onset, the gastrocnemius muscle from mice in each group were harvested and weighed. The muscle was then post-fixed in 4\% PFA in PBS for $30 \mathrm{~min}$ and then prepared for cryosectioning as described for the spinal cord. The muscle tissue was cryosectioned and incubated with chicken polyclonal antibody against
Neurofilament-H (1:500, Cat. No. AB5539, EMD Millipore) overnight at $4{ }^{\circ} \mathrm{C}$ in PBST $+10 \%$ goat serum. The tissues were then washed and incubated in appropriate secondary antibody and AlexaFluor 594-conjugated $\alpha$-bungarotoxin $(\alpha-\mathrm{BT})$ for $1 \mathrm{~h}$ at room temperature, washed again and coverslip mounted. Five images along the motor end plates in four muscle sections per mouse were collected and total and NF-H/ $\alpha-\mathrm{BT}$ co-labeled NMJs were quantified in Image J.

We plated NSC-34 cells transfected with human mSOD $1^{\text {G93A }}$ plasmid in 96-well plates for imaging of morphology and WST-1 viability (Roche) or lactate dehydrogenase $(\mathrm{LDH})$ release assays. Six-well culture plates were seeded and prepared for cell lysate collection and Western blot analysis. The cells were cultured in growth medium (Dulbecco's Modified Eagle Medium $[$ DMEM $]+10 \%$ fetal bovine serum (FBS, Hyclone) $+1 \%$ penicillin/streptomycin (Life Technologies) to approximately $80 \%$ confluence. Next, cells plated in both dish types were induced to express mutant SOD $1^{\mathrm{G} 93 \mathrm{~A}}$, and stress was applied through 24-h serum starvation (DMEM only). During starvation, cells were incubated with either saline (Control), $100 \mathrm{ng} / \mathrm{mL} \mathrm{bpV(pic)} \mathrm{(in} \mathrm{both}$ 96- and 6-well plates) or bpV(pic) plus $20 \mu \mathrm{g} / \mathrm{mL}$ PI3K inhibitor, LY294002, in DMEM to determine whether $\mathrm{bpV}$ (pic) acted through Akt signaling. The cell lysate was prepared in Laemmli sample buffer with 2-mercaptoethanol, loaded into 4-20\% sodium dodecyl sulfate (SDS) polyacrylamide TGX gels (Bio-Rad) for electrophoresis and Western blot analysis. Membranes were incubated overnight with rabbit monoclonal antibody against phospho-Akt (Ser473) (1:1000, Cell Signaling Inc., Cat. No. \#4060) and mouse monoclonal antibody against pan-Akt (1:1000, Cell Signaling Inc., Cat. No. 2920).

Cells in 96-well plates were washed in PBS, fixed with 4\% PFA, and imaged on an inverted brightfield microscope (Olympus CKX53). Formazan product from the WST-1 assay and colorimetric change from the LDH release assay were analyzed at 420 and $490 \mathrm{~nm}$, respectively, on a Bio-Tek plate reader. Statistical analysis was performed using GraphPad Prism 8.0 software (GraphPad, Inc). The normality of the data was determined using a Shapiro-Wilk test. Data between two groups were analyzed by a two-tailed unpaired Student's t-test, and data between multiple groups were analyzed by a one-way ANOVA and Newman-Keuls post hoc test. Group differences were considered statistically significant when $p<0.05$.

Quantification of MNs in the lumbar spinal cord ventral horn sections demonstrates the significant loss of MNs by day 90 with normal disease progression in mSOD1 ${ }^{\text {G93A }}$ mice (Fig. $1 \mathrm{~B}$ and D). Furthermore, both visual observation and quantitative analysis highlights 


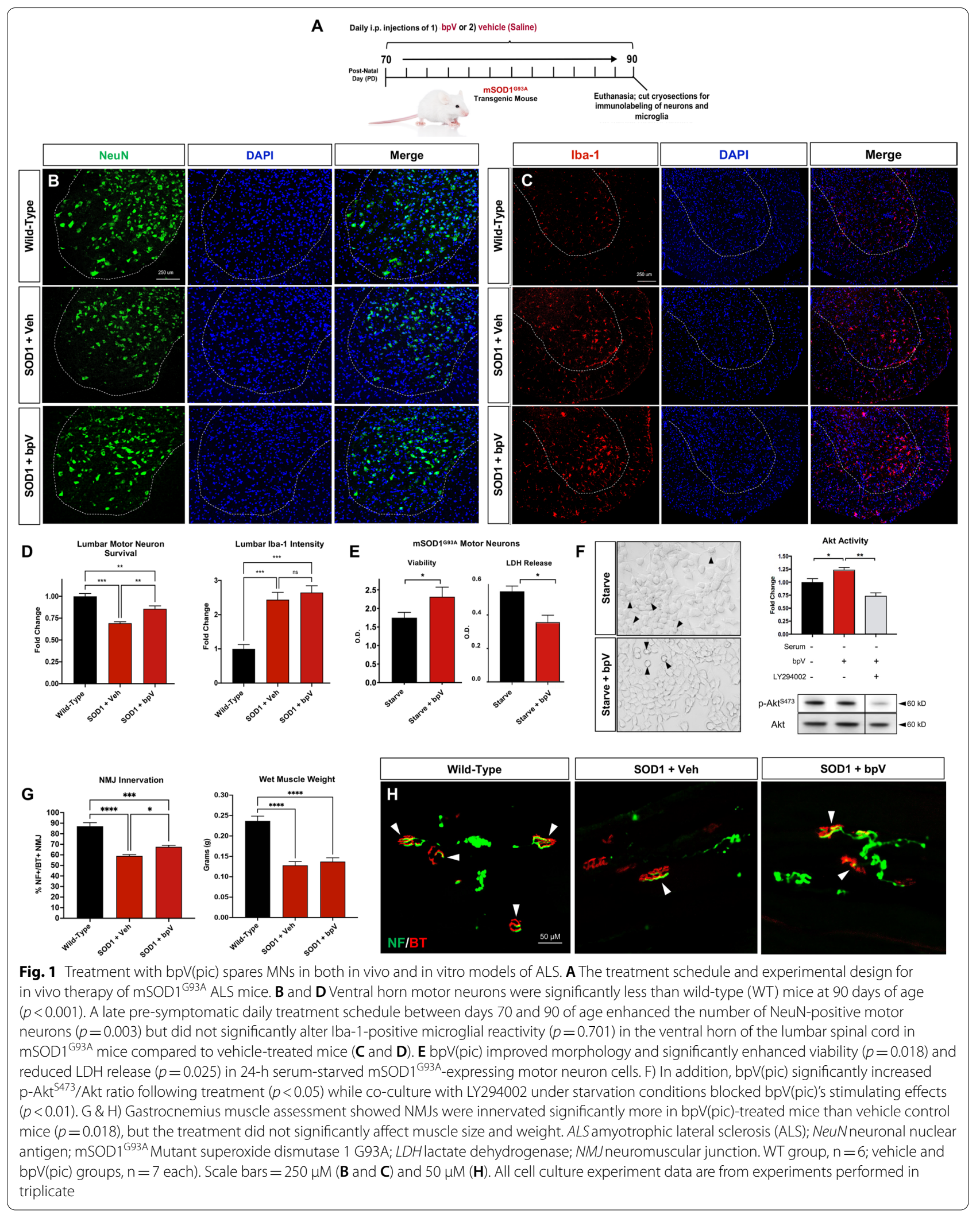


the significant reduction in $\mathrm{MN}$ loss following 20 days of systemic $\mathrm{bpV}$ (pic) treatment, especially in the most ventral region of the gray matter (Rexed Lamina IX). Recent work by Tung et al. [9] showed that dysregulation of miR17 in the $\mathrm{mSOD}^{\mathrm{G} 93 \mathrm{~A}}$ mouse leads to increased PTEN localization, which was associated with MN death and disease progression while replacing miR-17 prevented these effects. As such, it may be that PTEN phosphatase activity may only be part of PTEN's negative influence on MN survival, and dual targeting of PTEN expression/ localization and phosphatase activity could lead to even better outcomes. Given that the present study only targeted PTEN phosphatase activity, it cannot be ruled out that other functions of PTEN could be contributing to our observed results.

The $\mathrm{bpV}$ (pic) treatment paradigm did not exacerbate microglial activity in the ventral horn surrounding $\mathrm{MNs}$ (Fig. 1C and D), suggesting that either microglial reactivity is not detrimental to $\mathrm{MN}$ health at this stage in disease progression or that $\mathrm{bpV}(\mathrm{pic})$ may alter reactive microglia toward a more anti-inflammatory (M2) versus pro-inflammatory (M1) phenotype. Data support the latter, as PTEN exhibits a prohibitory effect on M1 to M2 macrophage transition [10]. We, however, did not examine M1 vs. M2 microglial phenotype to confirm whether PTEN inhibition by bpV(pic) modifies microglial phenotype in this way.

Our findings also indicated peripheral therapeutic effects of bpV(pic) therapy in mSOD1 ${ }^{\mathrm{G} 93 \mathrm{~A}}$ mice. Specifically, significantly more neuromuscular junctions (NMJs) were innervated in $\mathrm{bpV}(\mathrm{pic})$ treated mice compared to vehicle-treated controls (Fig. $1 \mathrm{G}$ and $\mathrm{H}$ ). It may be that central influence on $\mathrm{MN}$ soma as well as peripheral axonal and neuromuscular interaction were all imparted by the treatment. However, with building evidence of an increasingly underappreciated role of skeletal muscle on NMJ reinnervation and repair [11], it is possible the neuronal effects are secondary to target muscle alterations. When we measured wet weight of the gastrocnemius as a measure of treatment effects on muscle atrophy, no significant difference was found compared to vehicletreated mice (Fig. 1G). This suggests that metabolic changes in muscle may not have been significantly influenced by bpV(pic), but rather a combination of peripheral and central effects contributed to improved neuromuscular innervation. The present study did not investigate longer term effects on survival or function, which would aid in determining whether the increased innervation at 90 days of age leads to a delay of symptom progression and extension of lifespan.

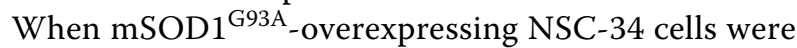
stressed with serum starvation for $24 \mathrm{~h}$ in the presence of $\mathrm{bpV}(\mathrm{pic})$, cell viability was significantly increased while cytotoxicity was significantly downregulated compared to control cells (Fig. 1E and F). As these findings indicate $\mathrm{bpV}(\mathrm{pic})$ imparts $\mathrm{MN}$ neuroprotection in both in vivo and in vitro $\mathrm{mSOD} 1^{\mathrm{G} 93 \mathrm{~A}} \mathrm{MNs}$, we anticipate that our results that PTEN inhibition and Akt activation in the $\mathrm{mSOD} 1^{\mathrm{G} 93 \mathrm{~A}}$ motor neuron cell line (Fig. 1F) is a likely mechanism of action for bpV(pic)'s protective effects observed in vivo. However, additional mechanistic investigation in the $\mathrm{mSOD} 1^{\mathrm{G} 93 \mathrm{~A}}$ mouse model will be required to test this hypothesis and validate our expected results.

This study is the first to demonstrate the therapeutic effects of $\mathrm{bpV}$ compounds through in vivo and in vitro experiments in $\mathrm{mSOD} 1^{\mathrm{G} 93 \mathrm{~A}}$ models of ALS. It must be noted that limitations concerning the time frame of investigation of disease status (pre-symptomatically only) and the relatively small sample size of the study impact broader implications of bpV(pic) therapy in altering disease course in later stages of disease. Nevertheless, our findings support prior reports that a primary mechanism of action of bpV(pic) is through inhibition of PTEN phosphatase activity and subsequent upregulation of downstream PI3K/Akt signaling $[12,13]$. With evidence mounting for a role of PTEN in MN degeneration in ALS, our evidence of neuroprotective and neuromuscular effects of $\mathrm{bpV}$ (pic) on MNs in ALS is promising and provides a new avenue of hope and research into potential effective therapies for preventing MN loss, paralysis, and death in ALS. With increasing support for neuroprotective and regenerative effects of PTEN inhibition, the more confidence that is established in $\mathrm{bpV}$ (pic) as a viable and valuable treatment option for ALS.

\section{Acknowledgements}

We thank the Androphy Lab at the Indiana University School of Medicine for kindly providing the $\mathrm{mSOD} 1^{\mathrm{G} 93 \mathrm{~A}}$-transfected NSC-34 cells.

\section{Authors' contributions}

JW provided conceptual input, performed experiments, analyzed data, and contributed to writing and editing the manuscript. LT performed histological experiments and subsequent data analysis and contributed to writing the manuscript. RM performed data analysis from cell culture experiments and prepared associated figures. TL performed histological experiments and associated data analysis. CW provided conceptual input, supervised the study, analyzed data, and wrote and edited the manuscript. All authors approve of the final manuscript.

\section{Funding}

This work was supported by funding from the United States Department of Veterans Affairs (Grant No. IK2RX002688) and Neurodegenerative Disease Research, Inc.

\section{Availability of data and materials}

Data from the current study are available from the corresponding author on reasonable request. 


\section{Declarations}

\section{Ethics approval and consent to participate}

All animal procedures were approved under the Guide for the Care and Use of Laboratory Animals (National Research Council) and the Guidelines of the Indiana University School of Medicine Institutional Animal Care and Use Committee.

\section{Consent for publication}

Not applicable.

\section{Competing interests}

No authors of the manuscript declare any conflicts of interest.

\section{Author details}

'Department of Biomedical Sciences and Comprehensive Care, Indiana University School of Dentistry, Indianapolis, IN 46202, USA. ${ }^{2}$ Neuromuscular Research Group, Richard L. Roudebush Veterans Affairs Medical Center, Indianapolis, IN 46202, USA.

Received: 6 July 2021 Accepted: 2 October 2021

Published online: 11 October 2021

\section{References}

1. Talbott EO, Malek AM, Lacomis D. The epidemiology of amyotrophic lateral sclerosis. Handb Clin Neurol. 2016;138:225-38.

2. Stopford MJ, Higginbottom A, Hautbergue GM, Cooper-Knock J, Mulcahy PJ, De Vos KJ, et al. C9ORF72 hexanucleotide repeat exerts toxicity in a stable, inducible motor neuronal cell model, which is rescued by partial depletion of Pten. Hum Mol Genet. 2017;26(6):1133-45.

3. Kirby J, Ning K, Ferraiuolo L, Heath PR, Ismail A, Kuo SW, et al. Phosphatase and tensin homologue/protein kinase B pathway linked to motor neuron survival in human superoxide dismutase 1-related amyotrophic lateral sclerosis. Brain. 2011;134(Pt 2):506-17.

4. Little D, Valori CF, Mutsaers CA, Bennett EJ, Wyles M, Sharrack B, et al. PTEN depletion decreases disease severity and modestly prolongs survival in a mouse model of spinal muscular atrophy. Mol Ther. 2015;23(2):270-7.
5. Walker CL, Walker MJ, Liu NK, Risberg EC, Gao X, Chen J, et al. Systemic bisperoxovanadium activates Akt/mTOR, reduces autophagy, and enhances recovery following cervical spinal cord injury. PLoS ONE. 2012;7(1):e30012.

6. Walker CL, Xu XM. PTEN inhibitor bisperoxovanadium protects oligodendrocytes and myelin and prevents neuronal atrophy in adult rats following cervical hemicontusive spinal cord injury. Neurosci Lett. 2014;573:64-8

7. Walker CL, Wu X, Liu NK, Xu XM. Bisperoxovanadium mediates neuronal protection through inhibition of PTEN and activation of PI3K AKT-mTOR signaling after traumatic spinal injuries. J Neurotrauma. 2019;36(18):2676-87.

8. Mancuso R, Olivan S, Mancera P, Pasten-Zamorano A, Manzano R, Casas $C$, et al. Effect of genetic background on onset and disease progression in the SOD1-G93A model of amyotrophic lateral sclerosis. Amyotroph Lateral Scler. 2012;13(3):302-10.

9. Tung YT, Peng KC, Chen YC, Yen YP, Chang M, Thams S, et al. Mir-17-92 confers motor neuron subtype differential resistance to ALS-associated degeneration. Cell Stem Cell. 2019;25(2):193-209.

10. Li N, Qin J, Lan L, Zhang H, Liu F, Wu Z, et al. PTEN inhibits macrophage polarization from M1 to M2 through CCL2 and VEGF-A reduction and NHERF-1 synergism. Cancer Biol Ther. 2015;16(2):297-306.

11. Pikatza-Menoio O, Elicegui A, Bengoetxea X, Naldaiz-Gastesi N, Lopez de Munain A, Gerenu G, et al. The skeletal muscle emerges as a new disease target in amyotrophic lateral sclerosis. J Pers Med. 2021;11(7):1.

12. Schmid AC, Byrne RD, Vilar R, Woscholski R. Bisperoxovanadium compounds are potent PTEN inhibitors. FEBS Lett. 2004;566(1-3):35-8.

13. Yu H, Shao J, Huang R, Guan Y, Li G, Chen S, et al. Targeting PTEN to regulate autophagy and promote the repair of injured neurons. Brain Res Bull. 2020;165:161-8.

\section{Publisher's Note}

Springer Nature remains neutral with regard to jurisdictional claims in published maps and institutional affiliations.
Ready to submit your research? Choose BMC and benefit from:

- fast, convenient online submission

- thorough peer review by experienced researchers in your field

- rapid publication on acceptance

- support for research data, including large and complex data types

- gold Open Access which fosters wider collaboration and increased citations

- maximum visibility for your research: over $100 \mathrm{M}$ website views per year

At $\mathrm{BMC}$, research is always in progress.

Learn more biomedcentral.com/submissions 